\title{
Correlated Rare Failure Analysis via Asymptotic Probability Evaluation
}

\author{
Jun $\mathrm{Tao}^{1}$, Handi $\mathrm{Yu}^{1}$, Dian Zhou ${ }^{* 1,2}$, Yangfeng Su${ }^{3}$, Xuan Zeng ${ }^{* 1}$, Xin $\mathrm{Li}^{* 4,5}$ \\ ${ }^{1}$ ASIC \& System State Key Lab, Dept. of Microelectronics, Fudan University, Shanghai, China, \\ ${ }^{2}$ Dept. of EE, University of Texas at Dallas, Dallas, USA, \\ ${ }^{3}$ School of Mathematical Sciences, Fudan University, Shanghai, China, \\ ${ }^{4}$ Dept. of ECE, Duke University, Durham, USA, \\ ${ }^{5}$ Institute of Applied Physical Sciences and Engineering, Duke Kunshan University, Kunshan, China
}

\begin{abstract}
In this paper, a novel Asymptotic Probability Estimation (APE) method is proposed to estimate the probability of correlated rare failure events for complex integrated systems containing a large number of replicated cells. The key idea is to approximate the failure rate of the entire system by solving a set of nonlinear equations derived from a general analytical model. An error refinement method based on Look-up Table (LUT) is further developed to improve numerical stability and, hence, reduce estimation error. Our numerical experiments demonstrate that compared to the state-of-the-art method, APE can reduce the estimation error by up to $45 \times$ without increasing the computational cost.
\end{abstract}

\section{INTRODUCTION}

The advance of integrated circuit (IC) technology brings about significant process variations, making robust circuit design a grand challenge. For instance, in order to achieve sufficiently high yield of an advanced microprocessor containing millions or even billions of replicated cells (e.g., SRAM bit-cells, flip-flops, etc.), the failure rate of each single cell must be extremely small. Considering a $1 \mathrm{Mb}$ SRAM array, the failure rate of one SRAM bit-cell must be less than $10^{-6}$ to ensure that the entire array can function properly with high yield. To accurately estimate such a rare failure rate, the traditional Monte Carlo (MC) method must repeatedly run expensive transistor-level simulations for more than $10^{7} \sim 10^{8}$ times and, therefore, is not computationally affordable.

A large body of works have been proposed in the literature to efficiently estimate rare cell-level failure rate [1] (i.e., the failure probability of a single cell). Most of them are based on Important Sampling (IS) [2]-[4] or Boundary Searching (BS) [5]. More recently, Scaled-Sigma Sampling (SSS) [6]-[8] and Subset Simulation (SUS) [9] are proposed to handle large-scale circuits characterized by a high-dimensional variation space involving hundreds of or even thousands of independent random variables.

Even though the problem of estimating cell-level rare failure rate has been extensively studied, it remains an open question how we could efficiently estimate the rare failure rate for an entire system (i.e., system-level failure rate [1]) containing a large number of replicated cells. The system-level failure events depend

\footnotetext{
* Corresponding authors: zhoud@utdallas.edu, xzeng@fudan.edu.cn, xinli.ece@duke.edu

Permission to make digital or hard copies of all or part of this work for personal or classroom use is granted without fee provided that copies are not made or distributed for profit or commercial advantage and that copies bear this notice and the full citation on the first page. Copyrights for components of this work owned by others than the author(s) must be honored. Abstracting with credit is permitted. To copy otherwise, or republish, to post on servers or to redistribute to lists, requires prior specific permission and/or a fee. Request permissions from Permissions@acm.org. DAC '17, June 18 - 22, 2017, Austin, TX, USA

Copyright is held by the owner/author(s). Publication rights licensed to ACM. ACM 978-1-4503-4927-7/17/06 ..\$15.00

DOI: http://dx.doi.org/10.1145/3061639.3062217
}

on a large number of cell-level failures. The entire system fails if any one of the cells fails. Note that cell-level failures are often statistically correlated due to the strong coupling at circuit or device levels, e.g., shared sense amplifier (SA) among multiple SRAM bit-cells [10], inter-die process variations [11]-[12], common power supply variations, etc. For this reason, when one cell fails, other cells tend to fail with high correlation. If all cell failures are modeled as statistically independent events and the correlation information is completely ignored, the system-level failure rate cannot be estimated accurately.

To address this correlation issue, Asymptotic Probability Approximation (APA) has been recently proposed [1]. It captures the correlation among cell-level failures by exploring a series of partial failure events (i.e., the probability for a small number of cells to fail). Based on the APA model, the system-level failure rate is simply a linear function of the total number of cells in the system.

In this paper, we further demonstrate that the system-level failure rate with correlated cell-level failure events is generally a nonlinear function of the number of cells. We propose a novel Asymptotic Probability Evaluation (APE) method to accurately capture the system-level failure rate by solving a set of nonlinear equations. Our key idea is to decouple the system-level failure into a set of statistically independent local failure events and fully correlated global failure events. Next, a general nonlinear analytical model is derived where the unknown model coefficients are solved by nonlinear optimization [13]. Finally, the failure rate of the entire system is estimated according to the aforementioned model.

Furthermore, in order to improve the numerical stability of our prosed method, an error refinement algorithm based on Lookup Table (LUT) is developed. It checks the numerical error based upon a set of inequalities derived from probability theorems. If the error is substantially large and, hence, the inequalities are violated, a correction step is applied to reduce the error. Such an error refinement process is particularly important when there exist a large number of replicated cells in the system and, therefore, the impact of numerical error can be significant. Compared to the traditional APA method [1], our proposed APE method can reduce the estimation error by up to $45 \times$ without increasing the computational cost, as will be demonstrated by the numerical results in Section 4.

The remainder of this paper is organized as follows. In Section 2, we derive the proposed APE algorithm and then discuss several important implementation issues in Section 3. The efficacy of APE is demonstrated by two experimental examples in Section 4. Finally, we conclude in Section 5.

\section{PROPOSED APPROACH}

\subsection{Problem Definition}

Suppose that we have a system containing $N$ replicated cells. Let $\mathbf{x}$ denote its device-level random variables (e.g., the threshold voltage $V_{T H}$, transistor widths and lengths, etc.) and $\left\{y_{n} ; n=1,2\right.$, 
$\cdots, N\}$ denote the performance of interest (PoI) of each cell (e.g., the read output of a single SRAM bit-cell). Without loss of generality, we assume that $\mathbf{x}$ can be divided into $N+1$ sub-vectors:

$$
\mathbf{x}=\left[\begin{array}{lllll}
\mathbf{x}_{l, 1}^{T} & \mathbf{x}_{l, 2}^{T} & \cdots & \mathbf{x}_{l, N}^{T} & \mathbf{x}_{g}^{T}
\end{array}\right]^{T},
$$

where the global variables $\mathbf{x}_{g}$ affect the PoI of all cells, but the $n$ th subset of local variables $\mathbf{x}_{l, n}$ impact the $n$-th cell only. For different cells, the value of $\mathbf{x} g$ is the same but the local variable values are different. We further assume that $\{\mathbf{x} l, n ; n=1,2, \cdots, N\}$ and $\mathbf{x}_{g}$ are mutually independent, after principal component analysis [14].

The aforementioned global and local variables do not necessarily correspond to inter-die and intra-die variations. To intuitively explain this concept, we consider the SRAM example in Figure 1. This SRAM array consists of $N$ bit-cells that share the same Sense Amplifier (SA). To read CELL $<1>$, we pre-charge the bit lines (i.e. $B L$ and $B L B$ ) and enable the corresponding word line $W L_{1}$. Next, CELL $<1>$ is selected and connected to both bit lines through the transistors $M_{3}$ and $M_{6}$. If the resulting differential voltage (i.e., $\Delta V_{B L}=V_{B L}-V_{B L B}$ ) is less than the input offset voltage of SA (i.e., $V_{O S}$ ), CELL $<1>$ cannot be read correctly and the read operation fails. Note that the read failure of each cell is determined by both the differential voltage $\Delta V_{B L}$ and the offset voltage $V_{O S}$. Sharing the same SA among all cells in the same column results in correlated read failures among these cells.

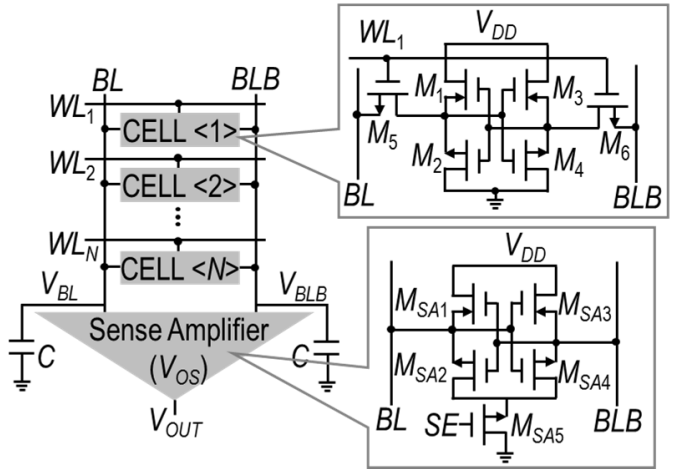

Figure 1. An example of SRAM array is shown with $N$ bit-cells and a sense amplifier.

In this case, we define the $n$-th local variables $\mathbf{x}_{l, n}$ as the local mismatches associated with the transistors in CELL $<n>$ and the global variables $\mathbf{x}_{g}$ as the local mismatches associated with the transistors $\left\{M_{S A 1}, M_{S A 2}, \cdots, M_{S A 5}\right\}$ in the SA. In addition, inter-die variations, power supply variations, temperature variations, etc. can also be defined as global variables since they affect all devices on the same die.

In general, the definition of global and local variables satisfies the following assumptions:

- When the global variables $\mathbf{x}_{g}$ are fixed, the failure events of different cells are only affected by their local variables $\{\mathbf{x} l, n ; n$ $=1,2, \cdots, N\}$ and, therefore, are manually independent, referred to as local failure events.

- When the local variables $\left\{\mathbf{x}_{l, n} ; n=1,2, \cdots, N\right\}$ are fixed, the failure events are only affected by the global variables $\mathbf{x}_{g}$ and, therefore, are fully correlated, referred to as global failure events.

With the aforementioned assumptions, we define the failure event of the $n$-th cell (i.e., $c_{n}$ ) as:

$$
c_{n}: y_{n}\left(\mathbf{x}_{l, n}, \mathbf{x}_{g}\right) \in F^{C},
$$

where $F^{C}$ represents the cell-level failure region in the performance space $U$ (i.e. the subset of the performance space where the PoI does not meet the specification). Sharing the global variables $\mathbf{x} g$ introduces correlations among the failure events of different cells. Since all cells are identically designed, we assume that the failure rates of different cells are identical:

$$
P^{C}=\operatorname{Pr}\left[y_{n}(\mathbf{x}) \in F^{C}\right]=\operatorname{Pr}\left[c_{n}\right],
$$

where $y_{n}(\mathbf{x})=y_{n}\left(\mathbf{x}_{l, n}, \mathbf{x}_{g}\right)$ and $\operatorname{Pr}[\bullet]$ stands for the probability that an event occurs. Note that the different behaviors posed by parasitics for different cells are ignored here.

If any cell fails, the SRAM system fails. Hence, the systemlevel failure event $F_{N}{ }^{U}$ can be defined as a union of all cell-level failure events [1], i.e., $F_{N}{ }^{U}=c_{1} \cup c_{2} \cup \cdots \cup c_{N}$. The system-level failure rate $P_{N}$ can be defined as:

$$
P_{N}=\operatorname{Pr}\left[\mathbf{y}(\mathbf{x}) \in F_{N}^{U}\right]=\operatorname{Pr}\left[c_{1} \cup c_{2} \cup \cdots \cup c_{N}\right],
$$

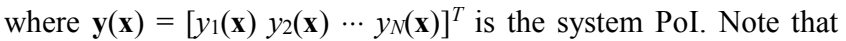
when $N$ is equal to $1, P_{1}$ equals the cell-level failure rate $P^{C}$.

\subsection{Asymptotic Probability Evaluation}

To estimate $P_{N}$, we introduce the $n$-th order simultaneous failure rate (SFR) $S_{n}$ as:

$$
S_{n}=\operatorname{Pr}\left[\mathbf{y}(\mathbf{x}) \in F_{n}^{S}\right]=\operatorname{Pr}\left[c_{1} \cap c_{2} \cap \cdots \cap c_{n}\right]=\operatorname{Pr}\left[\bigcap_{i=1}^{n} c_{i}\right] .
$$

$S_{n}$ denotes the probability for the $n$-th order simultaneous failure event $F_{n}{ }^{S}=c_{1} \cap c_{2} \cap \cdots \cap c_{n}$ to occur. Namely, it represents the probability for $n$ cells to fail simultaneously. $P_{N}$ can be rewritten as [1]:

$$
P_{N}=\sum_{n=1}^{N}(-1)^{n-1} \cdot\left(\begin{array}{l}
N \\
n
\end{array}\right) \cdot S_{n} .
$$

Note $S_{1}=P_{1}=P^{C}$. Theoretically, once $\left\{S_{n} ; n=1,2, \cdots, N\right\}$ are known, the system-level failure rate $P_{N}$ can be calculated by using (6). Hence, our goal is to accurately and efficiently estimate $\left\{S_{n} ; n\right.$ $=1,2, \cdots, N\}$.

The 1st order SFR $S_{1}$ can be expressed as the following integration over the entire region (i.e., $\Omega$ ) of the global variables $\mathbf{x}$ :

$$
S_{1}=\operatorname{Pr}\left[c_{1}\right]=\int_{\mathbf{x}_{g} \in \Omega} \operatorname{Pr}\left[c_{1} \mid \mathbf{x}_{g}\right] p d f\left(\mathbf{x}_{g}\right) d \mathbf{x}_{g},
$$

where $\operatorname{Pr}\left[c_{1} \mid \mathbf{x}_{g}\right]$ is the conditional probability of the failure event for the 1st cell given the global variables $\mathbf{x}_{g}$, and $p d f(\bullet)$ denotes the Probability Density Function (PDF) of a continuous random variable. We discrete the region $\Omega$ into a set of hyper-rectangles centered at $\left\{\mathbf{x}_{m}{ }^{\Omega} ; m=1,2, \cdots, M\right\}$ and approximate the integration in (7) by a finite number of summation terms:

$$
S_{1} \approx \sum_{m=1}^{M} \operatorname{Pr}\left[c_{1} \mid \mathbf{x}_{g}=\mathbf{x}_{m}^{\Omega}\right] p d f\left(\mathbf{x}_{g}=\mathbf{x}_{m}^{\Omega}\right) \Delta_{\mathbf{x}}=\sum_{m=1}^{M} l_{m} g_{m},
$$

where $\Delta_{\mathbf{x}}$ denotes the volume of a hyper-rectangle and $M$ is the approximation order. In (8), $l_{m}=\operatorname{Pr}\left[c_{1} \mid \mathbf{x}_{g}=\mathbf{x}^{\Omega}\right]$ is the failure rate of a single cell given $\mathbf{x}_{m}{ }^{\Omega}$ and it is referred to as the $m$-th local failure rate. On the other hand, $g_{m}=p d f\left(\mathbf{x}_{g}=\mathbf{x}_{m}{ }^{\Omega}\right) \cdot \Delta_{\mathbf{x}}$ is $m$-th global condition probability, i.e., the probability for the global variables $\mathbf{x}_{g}$ to fall into the $m$-th hyper-rectangle centered at $\mathbf{x}_{m} \Omega$. If the number of hyper-rectangles (i.e., $M$ ) is sufficiently large, we can obtain an accurate approximation for the integration in (7).

For the 2nd order SFR $S_{2}$ (i.e., the SFR of two cells), we can 
apply the similar approximation:

$$
\begin{aligned}
S_{2} & =\operatorname{Pr}\left[c_{1} \cap c_{2}\right]=\int_{\mathbf{x}_{g} \in \Omega} \operatorname{Pr}\left[c_{1} \cap c_{2} \mid \mathbf{x}_{g}\right] p d f\left(\mathbf{x}_{g}\right) d \mathbf{x}_{g} \\
& \approx \sum_{m=1}^{M} \operatorname{Pr}\left[c_{1} \cap c_{2} \mid \mathbf{x}_{g}=\mathbf{x}_{m}^{\Omega}\right] p d f\left(\mathbf{x}_{g}=\mathbf{x}_{m}^{\Omega}\right) \Delta_{\mathbf{x}}
\end{aligned}
$$

Note that given $\mathbf{x}^{\Omega}$, the failure event of the 1st cell (i.e. $c_{1}$ ) is only affected by the local variable $\mathbf{x}_{l, 1}$, and the failure event of the 2 nd cell (i.e. $c_{2}$ ) is only affected by the local variable $\mathbf{x}_{l, 2}$. Since $\mathbf{x}_{l, 1}$ and $\mathbf{x}_{l, 2}$ are mutually independent, the failure events of these two cells are also independent. The $m$-th local failure rate of two cells can be expressed as:

$$
\begin{aligned}
\operatorname{Pr}\left[c_{1} \cap c_{2} \mid \mathbf{x}_{g}=\mathbf{x}_{m}^{\Omega}\right] & =\operatorname{Pr}\left[\left(c_{1} \mid \mathbf{x}_{g}=\mathbf{x}_{m}^{\Omega}\right) \cap\left(c_{2} \mid \mathbf{x}_{g}=\mathbf{x}_{m}^{\Omega}\right)\right] \\
& =\operatorname{Pr}\left[c_{1} \mid \mathbf{x}_{g}=\mathbf{x}_{m}^{\Omega}\right] \operatorname{Pr}\left[c_{2} \mid \mathbf{x}_{g}=\mathbf{x}_{m}^{\Omega}\right]=l_{m}^{2}
\end{aligned}
$$

Substituting (10) into (9) yields:

$$
S_{2} \approx \sum_{m=1}^{M} l_{m}^{2} g_{m} .
$$

Similarly, the $n$-th order SFR (i.e., $S_{n}$ ) can be approximated by an analytical function of $l_{m}$ and $g_{m}$ :

$$
S_{n} \approx \sum_{m=1}^{M} l_{m}^{n} g_{m}
$$

Therefore, by introducing the global variable $\mathbf{x} g$ and a set of hyper-rectangles centered at $\left\{\mathbf{x}^{\Omega} ; m=1,2, \cdots, M\right\}$, the correlated failure events of different cells (i.e., $\left\{c_{n} ; n=1,2, \cdots, N\right\}$ ) can be decoupled into a set of independent local failure events (i.e., $\left\{c_{n} \mid\right.$ $\left.\left.\mathbf{x}_{g}=\mathbf{x}_{m}{ }^{\Omega} ; n=1,2, \cdots, N\right\}\right)$. If the $2 M$ variables $\left\{l_{m}, g_{m} ; m=1,2, \cdots\right.$, $M\}$ are known, all SFRs $\left\{S_{n} ; n=1,2, \cdots, N\right\}$ can be approximated by using (12) and then the system-level failure rate $P_{N}$ can be estimated by using (6). However, it is non-trivial to accurately estimate the conditional probabilities $\left\{l_{m} ; m=1,2, \cdots, M\right\}$, because the failure region associated with the 1 st cell (i.e. $c_{1}$ ) is not known in practice.

Instead of calculating the values of $\left\{l_{m} ; m=1,2, \cdots, M\right\}$ based on their definitions, we propose to take an alternative approach in this paper. The key idea is first to estimate a set of SFRs $\left\{S_{q} ; q=\right.$ $1,2, \cdots, Q\}$ by fast MC analysis (e.g., the H-SUS method [1]). Next, we solve the unknowns $\left\{l_{m} ; m=1,2, \cdots, M\right\}$ and $\left\{g_{m} ; m=1\right.$, $2, \cdots, M\}$ from the following nonlinear equations:

$$
\mathbf{L} \cdot \mathbf{g}=\mathbf{s} \text {, }
$$

where

$$
\mathbf{L}=\left[\begin{array}{cccc}
l_{1} & l_{2} & \cdots & l_{M} \\
l_{1}^{2} & l_{2}^{2} & \cdots & l_{M}^{2} \\
\vdots & \vdots & \ddots & \vdots \\
l_{1}^{Q} & l_{2}^{Q} & \cdots & l_{M}^{Q}
\end{array}\right], \quad \mathbf{g}=\left[\begin{array}{c}
g_{1} \\
g_{2} \\
\vdots \\
g_{M}
\end{array}\right], \quad \mathbf{s}=\left[\begin{array}{c}
S_{1} \\
S_{2} \\
\vdots \\
S_{Q}
\end{array}\right] .
$$

Three important clarifications should be made here. First, Eq. (13) is a set of nonlinear equations with $2 M$ unknown variables $\left\{l_{m} ; m=1,2, \cdots, M\right\}$ and $\left\{g_{m} ; m=1,2, \cdots, M\right\}$. We must know at least $2 M$ SFRs so that the equations in (13) are not underdetermined. Namely, $Q$ should be no less than $2 M$. Since the SFRs are extremely small, it is computationally expensive to estimate a large of SFRs in practice. Therefore, we must adopt a "low-order approximation" with small $M$ and $Q$ in order to make the computational cost affordable. As will be demonstrated by our numerical experiments in Section 4, even with an extremely small order (e.g., $M \leq 3$ ), our proposed method already offers superior accuracy over other state-of-the-art methods.

Second, once $l_{m}$ and $g_{m}$ are obtained by solving (13), $S_{n}$ can be estimated by using (12). We do not need to "explicitly" know $\left\{\mathbf{x}_{m} \Omega, m=1,2, \cdots, M\right\}$ that determine the locations of hyperrectangles. Conceptually, the optimal choice of these hyperrectangles is "implicitly" determined when solving (13). It is one of the most attractive features of the proposed method.

Third, if the nonlinear equations in (13) are neither underdetermined nor overdetermined (i.e., $Q=2 M$ ), the traditional AWE algorithm [15] can be used to solve (13) based on eigenvalue decomposition. However, due to the statistical error associated with $\mathrm{MC}$ analysis when estimating $\left\{S_{q} ; q=1,2, \cdots, Q\right\}$, forming a set of overdetermined nonlinear equations is often preferred for our application of interest. In this way, the solution of (13) is not strongly biased by the statistical errors. The aforementioned overdetermined equations can be solved by the following nonlinear optimization:

$$
\begin{array}{lll}
\min & \|\mathbf{L} \cdot \mathbf{g}-\mathbf{s}\|_{2} & \\
\text { s.t. } & 0 \leq l_{m} \leq 1 & (m=1,2, \cdots, M) . \\
& 0 \leq g_{m} \leq 1 \quad(m=1,2, \cdots, M)
\end{array}
$$

In (15), we aim to minimize the approximation error for $\left\{S_{q} ; q=\right.$ $1,2, \cdots, Q\}$ while guaranteeing that $0 \leq l_{m} \leq 1$ and $0 \leq g_{m} \leq 1$. Here, both $l_{m}$ and $g_{m}$ are within the interval $[0,1]$ because they represent probability values.

It is important to note that a number of implementation issues must be carefully addressed in order to make the proposed method of great efficiency. In the next section, we will further discuss these implementation details.

\section{IMPLEMENTAION DETAILS}

To estimate the extremely small SFRs $\left\{S_{q} ; q=1,2, \cdots, Q\right\}$, we adopt the Hierarchical Subset Simulation (H-SUS) method from [1]. First, according to the definition of the simultaneous failure event $F_{n}{ }^{S}$, we have:

$$
F_{q}^{S} \subseteq F_{q-1}^{S} \subseteq \cdots \subseteq F_{1}^{S}=F^{C} \subset F_{0}^{S}=U .
$$

The $q$-th order SFR (i.e., $S_{q}$ ) can be expressed as:

$$
\begin{aligned}
S_{q} & =\operatorname{Pr}\left[\mathbf{y}(\mathbf{x}) \in F_{q}^{S} \mid \mathbf{y}(\mathbf{x}) \in F_{q-1}^{S}\right] \cdot \operatorname{Pr}\left[\mathbf{y}(\mathbf{x}) \in F_{q-1}^{S}\right] \\
& =\prod_{i=1}^{q} \operatorname{Pr}\left[\mathbf{y}(\mathbf{x}) \in F_{i}^{S} \mid \mathbf{y}(\mathbf{x}) \in F_{i-1}^{S}\right]=\prod_{i=1}^{q} S_{i}^{C}
\end{aligned}
$$

where

$$
S_{i}^{C}=\operatorname{Pr}\left[\mathbf{y}(\mathbf{x}) \in F_{i}^{S} \mid \mathbf{y}(\mathbf{x}) \in F_{i-1}^{S}\right]
$$

denotes the failure probability of the $i$-th cell given that $i-1$ cells have simultaneously failed, and $S_{1}{ }^{C}$ is equal to the cell-level failure rate $P^{C}$

We define a set of intermediate failure events $\left\{F_{i, k} S ; k=1,2\right.$, $\left.\cdots, K_{i}\right\}$ that satisfy the following condition:

$$
F_{i}^{S}=F_{i, K_{i}}^{S} \subset F_{i, K_{i}-1}^{S} \cdots \subset F_{i, 1}^{S} \subset F_{i, 0}^{S}=F_{i-1}^{S},
$$

where $i \in\{1,2, \cdots, q\}, F_{i, 0} S=F_{i-1} S=F_{i-1, K_{i-1}}{ }^{S}$, and $F_{1,0} S=F_{0} S=U$. $S_{i}{ }^{C}$ can be further decoupled as the product of a set of conditional probabilities $\left\{S_{i, k}{ }^{C} ; k=1,2, \cdots, K_{i}\right\}$ :

$$
\begin{aligned}
S_{i}^{C} & =\operatorname{Pr}\left[\mathbf{y}(\mathbf{x}) \in F_{i, K_{i}}^{S} \mid \mathbf{y}(\mathbf{x}) \in F_{i, K_{i}-1}^{S}\right] \operatorname{Pr}\left[\mathbf{y}(\mathbf{x}) \in F_{i, K_{i}-1}^{S} \mid \mathbf{y}(\mathbf{x}) \in F_{i, 0}^{S}\right] \\
& =\prod_{k=1}^{K_{i}} \operatorname{Pr}\left[\mathbf{y}(\mathbf{x}) \in F_{i, k}^{S} \mid \mathbf{y}(\mathbf{x}) \in F_{i, k-1}^{S}\right]=\prod_{k=1}^{K_{i}} S_{i, k}^{C}
\end{aligned}
$$

where

$$
S_{i, k}^{C}=\operatorname{Pr}\left[\mathbf{y}(\mathbf{x}) \in F_{i, k}^{S} \mid \mathbf{y}(\mathbf{x}) \in F_{i, k-1}^{S}\right]
$$


Here, $F_{i, k} S$ and $K_{i}$ are usually determined experimentally. If we can find the proper events $\left\{F_{i, k}{ }^{S} ; k=1,2, \cdots, K_{i}\right\}, S_{i, k}{ }^{C}$ is substantially larger than $S_{q}$ and, hence, can be estimated efficiently by Markov Chain Monte Carlo (MCMC) sampling [9]. Next, $S_{q}$ can be efficiently calculated by using (20) and (17).

Once $l_{m}$ and $g_{m}$ have been found by solving the optimization problem in (15), we can calculate $\left\{S_{n} ; n=1,2, \cdots, N\right\}$ and $P_{N}$ by using (12) and (6) respectively. However, two important issues must be carefully considered in practice. First, if $N$ is large (i.e., there are many replicated cells), the binomial coefficients in (6) are extremely large and cannot be accurately represented by a double-precision floating-point number. Second, the statistical error posed by MC analysis often accumulates and substantially biases the estimated system-level failure rate as $N$ increases.

In order to address the aforementioned issues related to numerical stability, we propose a novel error refinement method based on LUT. Note that $P_{N}$ in (4) can be rewritten as:

$$
P_{N}=\operatorname{Pr}\left[c_{1} \cup c_{2} \cup \cdots \cup c_{N}\right]=P_{1}+P_{N-1}-\operatorname{Pr}\left[c_{1} \cap\left(\bigcup_{i=2}^{N} c_{i}\right)\right] .
$$

To simply our notation, we denote:

$$
T_{J, K}=\operatorname{Pr}\left[\left(\bigcap_{j=1}^{J} c_{j}\right) \cap\left(\bigcup_{k=1}^{K} c_{k}\right)\right] \quad(J, K \in\{1,2, \cdots, N-1\}),
$$

and $T_{1, N-1}$ as the last item at right-hand side of (22):

$$
T_{1, N-1}=\operatorname{Pr}\left[c_{1} \cap\left(\bigcup_{i=2}^{N} c_{i}\right)\right] .
$$

Based on (22)-(24), we have:

$$
\begin{aligned}
P_{2} & =P_{1}+P_{1}-T_{1,1} \\
P_{3} & =P_{1}+P_{2}-T_{1,2} \\
& \vdots \\
P_{N-1} & =P_{1}+P_{N-2}-T_{1, N-2} \\
P_{N} & =P_{1}+P_{N-1}-T_{1, N-1}
\end{aligned}
$$

Once $\left\{T_{1, K} ; K=1,2, \cdots, N-1\right\}$ are known, $P_{N}$ can be calculated by using (25).

For any $J$ and $K \in\{1,2, \cdots, N-1\}$, we have:

$$
\begin{aligned}
& \operatorname{Pr}\left[\left(\bigcap_{j=1}^{J} c_{j}\right) \cap\left(\bigcup_{k=1}^{K} c_{k}\right)\right]=\operatorname{Pr}\left[\bigcap_{j=1}^{J+1} c_{j}\right] \\
& \quad+\operatorname{Pr}\left[\left(\bigcap_{j=1}^{J} c_{j}\right) \cap\left(\bigcup_{k=1}^{K-1} c_{k}\right)\right]-\operatorname{Pr}\left[\left(\bigcap_{j=1}^{J+1} c_{j}\right) \cap\left(\bigcup_{k=1}^{K-1} c_{k}\right)\right]
\end{aligned}
$$

Eq. (26) can be re-written as:

$$
T_{J, K}=T_{J, 1}+T_{J, K-1}-T_{J+1, K-1} .
$$

Hence, we can construct a LUT T based on (27) to calculate $\left\{T_{1, K}\right.$; $K=1,2, \cdots, N-1\}$ :

$$
\mathbf{T}=\left[\begin{array}{lllll}
T_{1,1} & T_{1,2} & \cdots & T_{1, N-2} & T_{1, N-1} \\
T_{2,1} & T_{2,2} & \cdots & T_{2, N-2} & \\
\vdots & \vdots & . & & \\
T_{N-2,1} & T_{N-2,2} & & & \\
T_{N-1,1} & & & &
\end{array}\right],
$$

where $\mathbf{T}$ is a triangular matrix. Each item in $\mathbf{T}$ with $1 \leq J<N$ and $1<K \leq N-1$ satisfies (27).

To determine $\mathbf{T}$, we calculate each column based on (27) in sequence. Let us start from the first column with $K=1$. According to the definition of $T_{J, K}$ in (23), we have:

$$
T_{J, 1}=\operatorname{Pr}\left[\left(\bigcap_{j=1}^{J} c_{j}\right) \cap c_{1}\right]=S_{J+1},
$$

where $\left\{S_{J+1} ; J=1,2, \cdots, N-1\right\}$ have been already calculated according to (12). For the second column with $K=2$, each item
$T_{J, 2}$ is determined by those in the first column:

$$
T_{J, 2}=T_{J, 1}+T_{J, 1}-T_{J+1,1} \text {. }
$$

Next, based on (27), the remaining columns are calculated by using the previous column. The aforementioned procedure eliminates the need of directly computing the binomial coefficients in (6) and, hence, substantially improves the numerical stability.

The LUT $\mathbf{T}$ in (28) carries two important properties. First, since $\mathrm{U}_{k=1}{ }^{K} c_{k}$ is always a subset of $\mathrm{U}_{k=1}{ }^{K+1} c_{k}, T_{J, K}$ defined in (23) should increase with $K$ (i.e., $T_{J, K} \leq T_{J, K+1}$ ). In addition, it is straightforward to observe from (23) that $T_{J, K}$ should not be greater than $T_{J-1,1}$ (i.e., $T_{J, K} \leq T_{J-1,1}=\operatorname{Pr}\left[\cap_{j=1}^{J} c_{j}\right]$ ). Due to the statistical error posed by $\mathrm{MC}$ analysis, the aforementioned two inequalities $T_{J, K} \leq T_{J, K+1}$ and $T_{J, K} \leq T_{J-1,1}$ may be violated. Consequently, the iterative procedure of building the LUT T may become numerically unstable. To make the proposed approach numerically robust, if any of the two inequalities does not hold, we will reset $T_{J, K}$ to $\min \left(T_{J, K+1}, T_{J-1,1}\right)$. In other words, the value of $T_{J, K}$ is updated in order to force the two inequalities to hold.

Second, since $\bigcap_{j=1}^{J+1} c_{j}$ is always a subset of $\bigcap_{j=1}^{J} c_{j}$ as defined in (23), the values of $T_{J, K}$ in the same column should decrease with the increase of $J$ (i.e., $T_{J+1, K} \leq T_{J, K}$ ). From (27), we have

$$
T_{J+1, K}-T_{J, K}=T_{J, 1}-T_{J, K+1} \text {. }
$$

With the proposed error refinement technique, we can guarantee that $T_{J, K} \leq T_{J, K+1}$. Namely, the elements in the same row should increase with $K$ and, hence, we have $T_{J, 1} \leq T_{J, K+1}$ and $T_{J+1, K}-T_{J, K}$ $=T_{J, 1}-T_{J, K+1} \leq 0$.

Algorithm 1 summarizes the major steps of the proposed error refinement algorithm based on LUT. When we calculate $T_{J, K}$ from Step 7 to Step 10, the required elements $T_{J, 1}, T_{J, K-1}, T_{J+1, K-1}$ and $T_{J-1,1}$ have already been obtained during the previous iterations. Similarly, when we calculate $P_{n}$ in Step 15, $P_{n-1}$ has already been available. Algorithm 1 takes advantage of the LUT T to improve the numerical stability by applying the proposed error correction at Step 9.

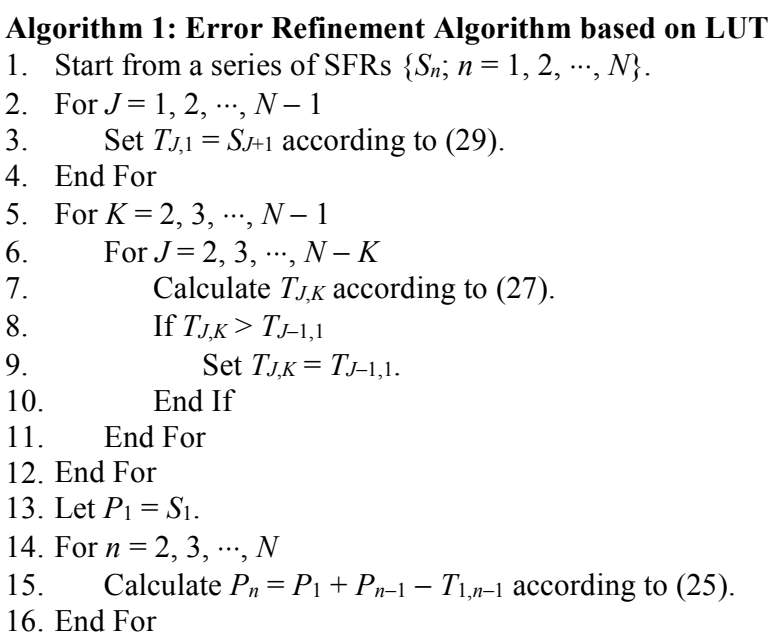

Algorithm 2 further summarizes the major steps of the proposed APE to estimate the system-level failure rate $P_{N}$. Since $P_{N}$ is calculated based on the nonlinear model (12), it is a nonlinear function of the number of cells (i.e., $N$ ). We can iteratively increase $Q$ until the estimations of $P_{N}$ obtained with $Q-1$ SFRs and $Q$ SFRs are almost identical. In practise, the order of APE (i.e., $M$ ) is usually set to the maximum integer that does 
not exceed $Q / 2$.

Algorithm 2: Asymptotic Probability Evaluation (APE)

1. Suppose that the number of required SFRs is $Q$, the order of the adopted SFR approximation (12) is $M$ (also referred to as the order of APE) and $Q \geq 2 M$.

2. Estimate the SFRs $\left\{S_{q} ; q=1,2, \cdots, Q\right\}$ by adopting H-SUS [1].

3. Calculate $l_{m}$ and $g_{m}$ by using SQP [13] or any other nonlinear programming method to solve the optimization problem in (15).

4. Calculate the SFRs $\left\{S_{n} ; n=1,2, \cdots, N\right\}$ based on (12).

5. Apply Algorithm 1 to estimate the system-level failure rate $P_{N}$.

\section{NUMERICAL EXPERIMENTS}

In this section, to demonstrate the efficacy of the proposed APE method, we estimate the read and write failure rates of an SRAM array shown in Figure 1. The SRAM array is designed with a 55nm CMOS technology and consists of $N \in\{1,2, \cdots, 64\}$ bit-cells. Three different approaches are implemented for comparison purpose: (i) the brute-force MC analysis, (ii) the APA method [1], and (iii) the proposed APE method. MC analysis is adopted to generate the "golden" system-level failure rate. All numerical experiments are performed on a computer cluster composed of 500 workstations and each workstation is equipped with $2.67 \mathrm{GHz} \mathrm{CPU}$ and $4 \mathrm{~GB}$ memory.

\subsection{Read Failure Rate}

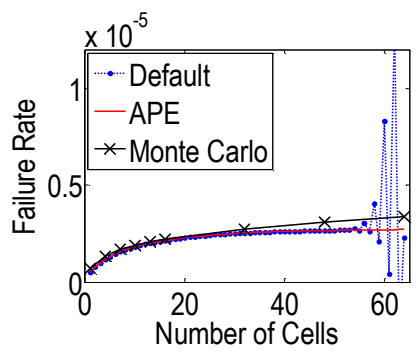

(a)

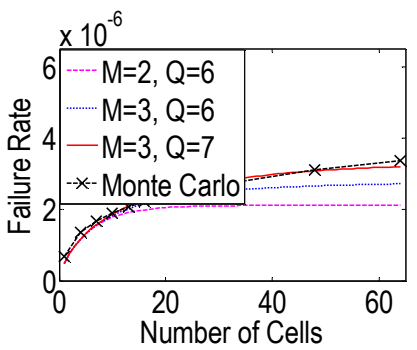

(b)
Figure 2. System-level read failure rates estimated by (a) different algorithms and (b) APE. The number of bit-cells (i.e., $N$ ) varies from 1 to 64 .

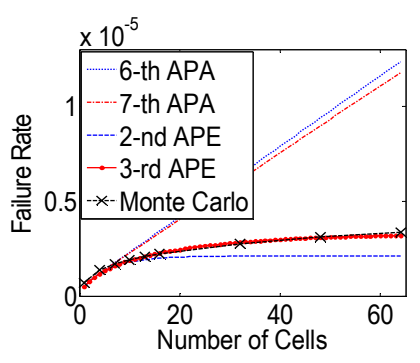

(a)

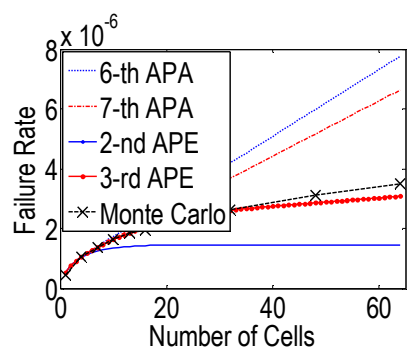

(b)
Figure 3. System-level failure rates estimated by the conventional APA method [1] and the proposed APE method: (a) read operation and (b) write operation.

When reading any cell from the SRAM array, if the differential bit-line voltage is smaller than the SA input offset voltage $V_{O S}$, the read operation fails. In this example, we do not consider the inter-die or power supply variations, but only take into account the $V_{T H}$ mismatches of all transistors in the circuit (i.e., all bit-cells and the SA) as independent random variables following Gaussian distributions. Hence, we have $6 \mathrm{~N}+5$ independent random variables in total. The number of random variables reaches 389 when $N$ equals 64 .

Figure 2(a) shows the system-level failure rates estimated by the nonlinear models (6) and (12) with $M=3$ and $Q=6$. The "Monte Carlo" results are obtained with $1.8 \times 10^{8}$ samples. We use H-SUS to estimate the SFR values $\left\{S_{1}, S_{2}, \cdots, S_{6}\right\}$. When applying the "Default" method, $l_{m}$ and $g_{m}$ are calculated by solving the optimization problem in (15) with SQP [13] and $P_{N}$ is calculated based on (6) directly. When the number of bit-cells increases (e.g., $N>55$ ), the estimated failure rates become numerically unstable, as shown in Figure 2(a). To address this issue, the proposed "APE" approach applies error refinement (i.e., Algorithm 1) to estimate $P_{N}$ and, consequently, the estimated failure rates become stable even when the number of cells is large.

Figure 2(b) shows the failure rates estimated by the proposed APE method with different orders (i.e., $M$ ) and SFR numbers (i.e., $Q$ ). When $Q$ is fixed as 6, a higher order APE (i.e., $M=3$ ) yields more accurate results than the lower order one (i.e., $M=2$ ). When the order $M$ is fixed as 3, APE with more SFRs (i.e., $Q=7$ ) achieves better accuracy than that with less SFRs (i.e., $Q=6$ ). Therefore, APE asymptotically approaches the actual failure rate as $M$ and $Q$ increase. In practice, APE with higher order and more SFRs is often preferred in order to improve estimation accuracy, if the computational cost is not prohibitively expensive.

Figure 3(a) further compares the failure rates estimated by APA and APE. Both the 6-th order APA and the 2-nd order APE apply H-SUS to obtain the same set of SFRs (i.e., $Q=6$ ) and, hence, almost share the same computational cost. Similarly, both the 7-th order APA and the 3-nd order APE also use the same set of SFRs (i.e., $Q=7$ ). Note that the failure rate estimated by APA increases linearly with the number of cells (i.e., $N$ ). Hence, its error becomes increasingly large when $N$ is large. On the other hand, the proposed APE method approximates the failure rate as a nonlinear function of $N$. Without increasing the computational cost, APE offers superior accuracy over APA. As shown in Figure $3(\mathrm{a})$, the 2-nd order APE is more accurate and less computationally expensive than the 7-th order APA in this example.

Table 1. Comparison between APA and APE for read failure rate estimation of a 64-bit SRAM array

\begin{tabular}{l|c|c|c}
\hline & \# of Samples & Estimated Failure Rate & Absolute Error \\
\hline 6-th APA & 7459 & $1.26 \mathrm{E}-05$ & $9.24 \mathrm{E}-06$ \\
\hline 7-th APA & 7631 & $1.18 \mathrm{E}-05$ & $8.44 \mathrm{E}-06$ \\
\hline 2-nd APE & 7459 & $2.51 \mathrm{E}-06$ & $8.50 \mathrm{E}-07$ \\
\hline 3-rd APE & 7631 & $3.19 \mathrm{E}-06$ & $1.70 \mathrm{E}-07$ \\
\hline
\end{tabular}

Table 1 compares the estimation results of APA and APE for our 64-bit SRAM array. The "golden" system-level read failure rate is estimated by MC analysis with $1.8 \times 10^{8}$ samples and is equal to $3.36 \times 10^{-6}$. The absolute error is defined as $\left|P_{M C}-P_{E S T}\right|$, where $P_{M C}$ denotes the "golden" failure rate estimated by $\mathrm{MC}$ and $P_{E S T}$ is the failure rate estimated by APA or APE. Compared to the 7-th order APA, the 3-rd order APE reduces the absolute error by $45 \times$ without increasing the computational cost (i.e., 7631 samples required for both methods). Even the 2-nd order APE with 7459 samples achieves higher accuracy than the 7-th order APA (about $10 \times$ reduction in absolute error).

\subsection{Write Failure Rate}

In this example, we study the Write Static Noise Margin (WSNM) of an SRAM array. As shown in Figure 4(a), a bit-cell 
can be divided into two inverters, i.e., InvQB and InvQ. We simulate the DC transfer function between Q and QB of these two inverters separately and form the butterfly curves in Figure 4(b). WSNM is defined as the width of the largest embedded square between the two butterfly curves. If the WSNM of one cell is less than a given threshold, we cannot correctly write it and, hence, cell-level write failure occurs. In this example, we model $V_{T H}$ variation of each transistor in the SRAM array by one inter-die random variable shared by all transistors and one independent intra-die random variable. All these random variables follow Gaussian distributions. In addition, the $V_{D D}$ variation is also taken into account and is modeled as a Gaussian distribution. The total number of random variables is $6 N+2$. It reaches 386 when $N$ equals 64 .

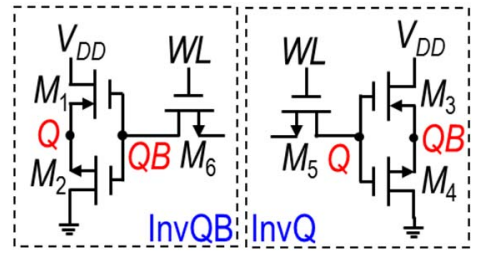

(a)

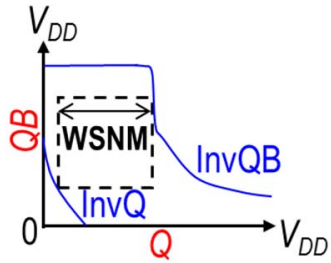

(b)
Figure 4. Write Static Noise Margin (WSNM) of a bit-cell: (a) two inverters InvQB and InvQ decomposed from a bit-cell, and (b) the DC transfer function of these two inverters.

Figure 3(b) shows the write failure rates estimated by APA and APE. The 6-th order APA and the 2-nd order APE share the same set of SFRs (i.e., $Q=6$ ), and the 7-th order APA and the 3nd order APE share the same set of SFRs (i.e., $Q=7$ ). In this example, APE again achieves superior accuracy over APA, without increasing the computational cost.

Table 2. Comparison between APA and APE for write failure rate estimation of a 64-bit SRAM array

\begin{tabular}{l|c|c|c}
\hline & \# of Samples & Estimated Failure Rate & Absolute Error \\
\hline 6-th APA & 7683 & $7.77 \mathrm{E}-06$ & $4.26 \mathrm{E}-06$ \\
\hline 7-th APA & 7996 & $6.64 \mathrm{E}-06$ & $3.13 \mathrm{E}-06$ \\
\hline 2-nd APE & 7683 & $1.44 \mathrm{E}-06$ & $2.07 \mathrm{E}-06$ \\
\hline 3-rd APE & 7996 & $3.08 \mathrm{E}-06$ & $4.30 \mathrm{E}-07$ \\
\hline
\end{tabular}

Table 2 compares the estimation results of APA and APE for write failure rate of the 64-bit SRAM array. The "golden" systemlevel write failure rate is estimated by MC analysis with $1.8 \times 10^{8}$ samples and is equal to $3.51 \times 10^{-6}$. Compared to the 7-th order APA, the 3-rd order APE reduces the absolute error by $7 \times$ without increasing the computational cost.

\section{CONCLUSIONS}

In this paper, we propose a novel Asymptotic Probability Estimation (APE) method to accurately estimate the probability of a large number of correlated rare failure events. APE approximates the system-level failure rate by constructing a general nonlinear model in terms of the total number of cells. An error refinement method based on LUT is further developed in order to improve the numerical stability and, hence, make the proposed APE method of practical utility. Our numerical experiments demonstrate that APE reduces the absolute error by up to $45 \times$ over the conventional APA method, without increasing the computational cost.

\section{ACKNOWLEDGEMENTS}

This research is supported partly by National Natural Science Foundation of China (NSFC) research project 61376041, $61376040,61574046,61574044$, and 91330201 , partly by the National Key Research and Development Program 2016YFB0201304, partly by the Thousand Talents Plan, partly by National Science Foundation (NSF) research project 1115556 and CCF-1604150, partly by the Laboratory of Mathematics for Nonlinear Science at Fudan University.

\section{REFERENCES}

[1] H. Yu et al., "Efficient statistical analysis for correlated rare failure events via asymptotic probability approximation," ICCAD, Nov. 2016.

[2] L. Dolecek et al., "Breaking the simulation barrier: SRAM evaluation through norm minimization," ICCAD, pp. 322-329, Nov. 2008

[3] K. Katayama et al., "Sequential importance sampling for lowprobability and high dimensional SRAM yield analysis," ICCAD, pp. 703-708, Nov. 2010.

[4] R. Kanj et al., "Mixture importance sampling and its application to the analysis of SRAM designs in the presence of rare failure events," $D A C$, pp. 69-72, Jul. 2006.

[5] A. Singhee et al., "Statistical blockade: very fast statistical simulation and modeling of rare circuit events, and its application to memory design," IEEE Trans. on CAD, vol. 28, no. 8, pp. 1176-1189, Aug. 2009.

[6] S. Sun et al., "Efficient SRAM failure rate prediction via Gibbs sampling," IEEE Trans. on CAD, vol. 31, no. 12, pp. 18311844, Dec. 2012.

[7] S. Sun et al., "Fast statistical analysis of rare circuit failure events via Bayesian scaled-sigma sampling for high-dimensional variation space," CICC, Sep. 2015.

[8] S. Sun et al., "Fast statistical analysis of rare circuit failure events via Scaled-Sigma sampling for high-dimensional variation space," IEEE Trans. on CAD, vol. 34, no. 7, pp. 1096-1109, June. 2015.

[9] S. Sun et al., "Fast statistical analysis of rare circuit failure events via subset simulation in high-dimensional variation space," ICCAD, pp. 324-331, Nov. 2014.

[10] E. I. Vatajelu et al., "Analyzing the effect of concurrent variability in the core cells and sense amplifiers on SRAM read access failures," IEEE ICDTIS, pp. 39-44, March 2013.

[11] S. Mukhopadhyay et al., "Modeling of failure probability and statistical design of SRAM array for yield enhancement in nanoscaled CMOS," IEEE Trans. on CAD, vol. 24, no. 12, pp. 1859-1880, Dec. 2005.

[12] M. Alioto et al., "Variations in nanometer CMOS Flip-Flops: part I-impact of process variations on timing," IEEE Trans. on CAS-I: Regular Papers, vol. 62, no. 8, pp. 2035-2043, Aug. 2015.

[13] J. Nocedal et al., Numerical Optimization, Springer, 2006.

[14] C. Bishop, Pattern Recognition and Machine Learning, Prentice Hall, 2007.

[15] L. Pillage et al., "Asymptotic waveform evaluation for timing analysis," IEEE Trans. on CAD, vol. 9, no. 4, pp. 352-366, Apr. 1990.

[16] H. Makino et al., "Accelerated evaluation method for the SRAM cell write margin using word line voltage shift," ISIC, pp. 63-66, Dec. 2011. 